\section{Animal welfare evaluation at a slaughterhouse for heavy pigs intended for processing}

\author{
Roberta Stocchi, ${ }^{1}$ \\ Nicholas Aconiti Mandolini, 2 \\ Maria Marinsalti, ${ }^{3}$ Natalina Cammertoni, ${ }^{1}$ \\ Anna Rita Loschi, ${ }^{1}$ Stefano Rea ${ }^{1}$ \\ 'Scuola di Bioscienze e Medicina \\ Veterinaria, Università degli Studi di \\ Camerino); \\ 2Scuola di Specializzazione in Sanità \\ Animale, Allevamento e Produzioni \\ Zootecniche, Università degli Studi di \\ Camerino; ${ }^{3}$ Scuola di Specializzazione in \\ Igiene e Controllo \\ dei Prodotti della Pesca e \\ dell'Acquacoltura, Università degli Studi \\ di Camerino, Matelica (MC), Italy
}

\section{Abstract}

The Council Regulation (EC) No. 1099/2009 requires slaughterhouse managers to implement specific standard operating procedures for all pre-slaughter stages considered at risk, aimed at achieving adequate levels of animal welfare. This survey was aimed at testing the applicability to an abattoir for heavy pigs of an assessment system of animal welfare through animal-based measures. In the monitoring of handling operations, the number of animals fallen/slipped and prodded, and that of vocalising pigs were recorded. In the monitoring of the immobilisation stage, carried out on the same pigs, vocalisations were recorded at the entrance to the box and falls/slips occurring inside it. Animal welfare assessment during the stunning-sticking-bleeding steps, was carried out by recording the head-only electrical stunning basic parameters set by legislation, vocalisations resulting from hot wanding, and clinical signs of consciousness, sensibility and certain death. Except for immobilisation, the percentage of occurrence of these events above acceptability limits was detected in all other preslaughter steps. The most critical stages were: handling in the unloading area and along the single-file chute, stunning and especially bleeding, where $84.13 \%$ of animals showed one or more signs of consciousness and/or sensibility recovery. Wrong placement of electrodes observed in $53.98 \%$ of the animals, insufficient voltage and low amperage may explain why a high percentage of pigs recovered consciousness and/or sensibility before death. Some simple restructuring of unloading area, slowdown of slaughter line speed, increase of personnel involved in pre-slaughter management and regular calibration of the electrical stunning device could be effectively corrective measures aimed at raising the animal welfare level at the slaughterhouse under study.

\section{Introduction}

During the last twenty years the European Community has issued a series of directives and horizontal and vertical regulations, which seek to ensure the welfare of livestock animal species in all steps of production, from breeding to slaughter, including transportation. The Council Regulation (EC) No. 1099/2009 (European Commission, 2009) on the protection of animals at the time of killing, in force as of January $1^{\text {st }} 2013$, is the current reference standard for those who work in slaughterhouses. The main novelty of the Regulation, which from 8 December 2019 will completely repeal the Council Directive No. 93/119/EEC (European Commission, 1993), is represented by the transfer of full responsibility for the protection of animal welfare to business operators (B0s), which must apply specific HACCP plans, similar to those implemented to ensure food safety. In particular, operators of slaughterhouses will have to develop and implement riskbased standard operating procedures (SOPs), able to ensure that, during the killing and related operations, such as handling, lairaging, restraining, stunning and bleeding, the animals are spared any pain, distress and unnecessary suffering. These procedures should include clear objectives, responsibilities, modus operandi, measurable criteria, as well as monitoring and recording procedures. In particular, as regards the stunning, the B0 has the obligation to establish a representative sample of animals to check that they do not show signs of consciousness and sensibility in the period between the end of the stunning process and death. To enable companies to comply with the provisions of the new legislation, the EU will identify indicators of animal welfare as regards killing and related operations that are easily monitored within the slaughterhouses, and critical limits which meet the EU standards. The assessment of animal welfare is generally carried out applying two types of parameters: structural measurements (engineering measures) and assessments made directly on animals (animal-based measures). The former evaluate the adequacy of facilities and equipment, the latter the response and/or the effect of the environment and/or management practices on animals. It is precisely toward the animal-based measures that the EFSA, appointed by the European Commission to draft guidelines to harmonise the procedures for checking and monitoring the welfare of the different species of livestock animals, is turning its attention to (European Food Safety Authority, 2012). A sys-
Correspondence: Roberta Stocchi, Scuola di Bioscienze e Medicina Veterinaria, Università degli Studi di Camerino, via Circonvallazione 93/95, 62024 Matelica (MC), Italy.

Tel. +39.073.7403444 - Fax: +39.073 .7403402$

E-mail: roberta.stocchi@unicam.it

Key words: Pig welfare, Slaughterhouse, Animalbased measures, Animal handling, Only-head electrical stunning.

Received for publication: 13 May 2013 Revision received: 25 September 2013. Accepted for publication: 26 September 2013.

This work is licensed under a Creative Commons Attribution 3.0 License (by-nc 3.0).

CCopyright R. Stocchi et al., 2014

Licensee PAGEPress, Italy

Italian Journal of Food Safety 2014; 3:1712

doi:10.4081/ijfs.2014.1712

tem based on measurements carried out directly on animals has been successfully used for a long time on a voluntary basis by major U.S. restaurant chains for audit activities on animal welfare at the slaughterhouse, aiming at the selection of suppliers (Grandin, 2012a). This system, developed by Temple Grandin for the American Meat Institute (AMI), takes into account the following five numerically scored key-criteria with specific limits of acceptability and specific classes of judgment: i) percentage of fallen animals during the handling; ii) percentage of animals moved with an electric prod; iii) percentage of animals that vocalise during handling and stunning; iv) percentage of animals stunned effectively at the first attempt; v) percentage of animals that remain insensitive during bleeding (Grandin, 2010, 2012b, 2013). The purpose of this investigation was to test the applicability of the above-mentioned system of assessment of animal welfare during handling and only-head electrical stunning at a slaughterhouse for heavy pigs intended for processing.

\section{Materials and Methods}

This survey was conducted by 3 observers in the a slaughterhouse area of a pork meat-curing factory in the province of Ancona (Italy), where 120-130 pigs per week with average live weight of $170 \mathrm{~kg}$ are slaughtered. The company carries out slaughtering activities 2 hours a week. It has the following facilities: an unloading platform; a lairage stall of $137 \mathrm{~m}^{2}$ with 9 pens; a single-file chute with a pneumatic guillotine gateway for transfer to the slaughter room, consisting of a straight ramp with a spray-sprinkling device for humidifying the skin of pigs and a floating raised curved tunnel (CP-0110; Mancini 
Spa, Amandola (AP), Italy); a stun box with a pneumatic guillotine gateway and a pneumatic rotating side wall (CP-0110; Mancini Spa); a conveyor belt for the sticking (RIRE-0055; Mancini Spa) and bleeding (TAPP, NDT-050; Mancini Spa). The different batches of pigs are usually conferred on Saturday morning and slaughtered the following Monday, after a 42-45 hour rest. Plastic bags are generally used for their handling and electrical prods (RobSet ${ }^{\circledR}$ Color; Plast Micron, Modena, Italy), when necessary. Stunning is carried out by means of tongs (TL002; Gozlin, Modena, Italy) connected to an electronic stunner (TE002; Gozlin) which provides $50 \mathrm{~Hz}$ sinusoidal alternating current. Two operators are involved in the pre-slaughter stages: one is responsible for animal handling and the other for restraining, stunning, sticking and bleeding. Due to the small number of observers and to the structural characteristics of the slaughterhouse which made it impossible to follow the animals during handling at an adequate distance, it was necessary to carry out the monitoring in 3 days from different fixed observation points. The first and second observations, carried out on 121 pigs, involved the animal handling from the unloading platform to the lairage entrance, and the handling of the same pigs to the slaughter room through the singlefile chute and the immobilisation step, respectively; finally, the third, performed on 126 subjects, involved the stunning, sticking and bleeding stages. The limited space of the lairage area and the lack of passing lanes reserved for the operators did not allow the pen filling and emptying steps to be observed, nor the monitoring of recovered animals. The assessment of animal welfare during handling was carried out by recording the number of fallen/slipped and prodded animals in two different check lists. Limited to the handling along the single-file chute, the number of vocalising pigs and the time the pigs waited in the chute were recorded on a specific check list. The number of vocalising pigs at the entrance and the number of fallen/slipped animals inside the stun box were recorded in two different check lists to assess the animal welfare during immobilisation. The waiting time inside the stun box before the application of tongs was also recorded. As reported by Grandin (Grandin, 2012b), the sudden loss of the upright position in which a part of the body other than the limbs touches the ground, was considered as a fall, an extended sound of both high amplitude and high frequency produced with an open mouth (squeal) exclusively determined by operators or equipment was recorded as a vocalisation, and the touching of the body with an electric prod (whether energised or not) was counted as an electrostimulation. The narrow space of the stunningsticking-bleeding area and the short stun-tostick interval did not allow all clinical signs of effective stunning immediately after the release from the stun box to be observed. Therefore, an observer positioned on the stun-sticking platform recorded the basic parameters of onlyhead electrical stunning, such as correct electrode placement, voltage and amperage, exposure time and stun-to-stick interval. Other parameters detectable at a distance, such as vocalisations due to hot wanding (use of prematurely energised tongs), onset of clonic seizures (pédalage), and further stunning attempts before sticking. Only the electrodes applied symmetrically on the temporal region or on either the hollow behind the ears, or as well as asymmetrically at the top and the bottom of the head were considered correctly placed (Grandin, 2012b). Another observer positioned on the bleeding platform recorded the number of sticked pigs during tonic seizure and the number of pigs that immediately after sticking and during the first $2 / 3$ of bleeding showed the following signs considered as indicators of effective stunning and consciousness/sensibility recovery by EFSA expert (EFSA, 2004): pédalage, fixed gaze, mydriasis, eyeball rotation, pupil, cornea and eyelid reflexes, nystagmus, pain reflex determined by picking the snout (nose pick), rhythmic breathing, vocalisation and attempts to raise. Finally, a third observer positioned at the end of the bleeding platform, recorded the time interval between sticking and immersion into the scalding tank, and some signs of certain death, such as complete muscle relaxation and absence of breathing. The noise produced by the equipment in the slaughter room and the vibrations of the conveyor belt did not allow the absence of heartbeat to be verified, while horizontal bleeding made the lingual ptosis difficult to be detected.

\section{Results}

The most frequent event observed during handling in the unloading area was the use of electric prod, observed in $9.09 \%$ of pigs examined, followed by the slip (6.61\%) and the fall (2.48\%). The monitoring of the handling in the single-file chute, during which there was no fall/slip, showed a high percentage of prodded (90.91\%) and vocalising (52.89\%) pigs and an average waiting time of 107 " ( \pm 60 ”). During immobilisation, whose average time was 26 " $( \pm 19 ")$, noticeable events were not recorded, except for a pig which vocalised upon entering the stun box $(0.83 \%)$. As regards the monitoring of stunning a correct positioning of the tongs was observed only in $46.02 \%$ of pigs. Particularly, in most animals the electrodes were placed symmetrically behind the mandible's corner (47.67\%), on either the hollow behind the ears $(25.39 \%)$ or on the temporal region (20.63\%). In a limited number of subjects the tongs were placed symmetrically on the dorsal part of the neck (3.97\%), on the cheeks close to the snout (1.59\%) and on the ears $(0.79 \%)$. In two pigs where electrodes were placed on the cheeks a further stunning was made by placing the electrodes on the temporal region. The basic current parameters showed values between 192 and $220 \mathrm{~V}$ (average $210.18 \pm 5.13$ ), and between 0.67 and $1.73 \mathrm{~A}$ (average $1.17 \pm 0.20)$. Most animals (94.44\%) were exposed to a current exceeding $200 \mathrm{~V}$, $22.31 \%$ received a current equal to or greater than $1.30 \mathrm{~A}$, the minimum level required by Council Regulation (EC) No. 1099/2009 (European Commission, 2009), 56.20\% between 1.00 and $1.29 \mathrm{~A}$, and the remaining $21.49 \%$ less than $1.00 \mathrm{~A}$. The exposure time was between 4 and 8 ". Vocalisations due to hot wanding were detected in $14.28 \%$ of pigs examined. The stun-to-stick interval was between 6 and 26". Most pigs (72.22\%) were sticked within 15" from stunning, $26.20 \%$ within 18 " and only two animals (1.58\%) after more than 20 ". From the notes reported by the observer in the relevant check list it was possible to relate the prolongation of stun-to-stick interval over 15" to the need of manual release of the conveyor belt by the stunning operator. Pédalage was observed in $44.44 \%$ and $98.41 \%$ of the animals examined before and after sticking, respectively. Immediately after sticking, in most animals (88.09\%) the eyes were closed. Fifteen pigs showed open eye with rotated eyeball $(10.31 \%)$ or fixed gaze and mydriasis (1.59\%). The analysis of the crosscheck lists of the first and second observer showed that these two animals were sticked after more than 20" from the end of the tong application. Soon, all pigs with closed eyelids opened their eyes, allowing the eyeball to be observed, which appeared rotated in $86.51 \%$. As regards the clinical signs of consciousness and sensibility, $84.13 \%$ of pigs showed both corneal and blink reflex, associated in four subjects $(3.17 \%)$ to pupil reflex and nystagmus, $80.16 \%$ showed rhythmic breathing, $30.16 \%$ reaction to nose pick, $10.32 \%$ vocalisation and $4.76 \%$ attempts to raise. In all subjects such signs appeared after variable times during the first $2 / 3$ of bleeding, with the exception of two pigs in which the signs were observed immediately after sticking. Despite the observation of a large number of animals with one or more signs of consciousness and sensibility, a second stunning was performed only on those pigs which attempted to raise immediately after sticking (1.59\%) or during bleeding (3.17\%). It is noteworthy that two of four pigs stunned for the second time during bleeding did not show pédalage and eyeball rotation, while pupil reflex and nystagmus were observed in all of them. Finally, at the end of bleeding, whose average duration was 254 " $( \pm 32 ")$, all animals showed complete muscle relaxation and absence of breathing. 


\section{Discussion}

In all pre-slaughter stages, frequencies of occurrence of one or more indicators of poor animal welfare above the acceptable limits defined for swine by Grandin (Grandin, 2012b) were recorded, with the exception of the immobilisation step. Particularly, as regards the movement in the unloading area, the fall was the only parameter showing a slightly higher frequency than the critical limit proposed by the same author. Almost all falls and slips, the latter considered as a secondary criterion for which there is no specific acceptability limit, occurred near the entrance of the lairage; here some structural weaknesses, such as the limited width of the door, which requires pigs to form a single line, the smooth floor and the light/shadow contrast resulting in poor indoor lighting especially on sunny days, have been identified. Therefore, it should be necessary to restructure the unloading area in order to allow the animals to walk side by side, to maintain floors in such a way to minimise the risk of slipping, falling and injuring, and to remove distractions resulting from the light/shadow contrast. All these structural requirements are foreseen in Council Regulation (EC) No. 1099/2009 and the first two aspects also in Council Directive No. 93/119/EEC (European Commission, 2009, 1993). During the handling in single-file chute, the percentage of prodded animals and those vocalising were the only two criteria that exceeded the limits of acceptability, grading both as serious problem (Grandin, $2012 \mathrm{~b})$. The lack of fallen/slipped animals testifies the structural suitability of the single-file chute, designed and built for the exclusive handling of heavy pigs. Whereas, the high variability of waiting times in the chute due to irregular animal flow to the slaughter room seems to be the main cause of the large number of prodded and vocalising pigs. In fact, the discontinuity of animal handling operations from the lairage boxes to the stun box led some groups of pigs to long waiting times in the single-file chute, improperly used as a sort of waiting pen. The animals repeatedly moved forward and backward, and overlapped, so most of them refused to enter at the opening of the stun box's door, forcing the operator to frequently use the electric prod. It must be emphasised that a steady supply of animals from the holding pens to the slaughter room is explicitly required by Council Regulation (EC) No. 1099/2009 (European Commission, 2009). Handling small groups of animals, slowing the speed of the slaughter line or, alternatively, increasing the personnel involved in the preslaughter stages are suggested as corrective measures to raise the level of animal welfare during this step. These measures would also reduce the waiting time in the stun box, too long for some subjects. Indeed, as noted in the check list used for monitoring the immobilisation step, all the delays in the application of electrodes resulted from the need of manual release of the conveyor belt by the stunning operator due to the irregular pig flow. As regards stunning, animalbased measures indirectly used to assess the effectiveness of stunning, such as the percentage of vocalisations due to hot wanding and the percentage of correct positioning of the tongs, were considerably higher and lower, respectively, than the limits of acceptability, scoring both as serious problem (Grandin, 2012b). The frequent wrong placement of the electrodes seems to be the main factor affecting the high percentage of vocalisations due to hot wanding and the percentage of pigs showing signs of consciousness and/or sensitivity recovery during bleeding; according to this criterion, the presence of only one sensitive subject out of 100 animals checked is sufficient to score the slaughterhouse as not acceptable (Grandin, 2012b). In fact, the positioning of the electrodes in different areas from those considered ideal implies a remarkable increase of the impedance; this increase hinders the current flow and may delay or prevent the achievement of minimum amperage necessary to induce an effective and enduring stunning, leading to possible painful electric shocks and consciousness and/or sensitivity recovery before the death (EFSA, 2004). Even a malfunctioning of the stunning device may have contributed to the high percentage of conscious and/or sensitive pigs during bleeding. Actually, the voltage was set to $245 \mathrm{~V}$ but it never exceeded $220 \mathrm{~V}$, well below the recommended minimum value of $250 \mathrm{~V}$ (Anil and McKinstry, 1998; EFSA, 2004; Grandin, $2012 \mathrm{~b})$. Therefore, effective corrective measures aimed at raising the level of animal welfare at this critical pre-slaughter stage may be represented by increasing the number of operators in the stun-to-stick area or, alternatively, by slowing down the speed of the slaughter line, in order to enable the stunner to pay more attention to the positioning of tongs, as well as by regularly calibrating the stunning device. Finally, it must be emphasised that the EFSA has recently urged a public panel discussion among all stakeholders to better define the indicators of effective stunning and of consciousness and/or sensitivity recovery. The observed frequencies suggest that in the electrical stunning of pigs by means of tongs followed by horizontal bleeding the pédalage, easily detectable even at a distance, is the best indicator of effective stunning, and that the corneal and eyelid reflexes as well as rhythmic breathing are the best indicators of sensitivity recovery after sticking.

\section{Conclusions}

The results obtained at the slaughterhouse under investigation demonstrate the applicability and especially the usefulness of the animal welfare assessment system developed by Grandin for AMI. Besides allowing the steps at greater risk to be identified, the animal-based measures allowed the causes of non-compliance with animal welfare to be determined and the corrective measures to be suggested. The main advantage of this system is represented by the small number of criteria considered, which makes the monitoring of pre-slaughter stages, particularly handling, easier. Some concern arises about the acceptability limits defined by the same author on the basis of standards guaranteed by US industrial slaughterhouses which may be too restrictive for our production realities. In fact, especially light pigs are slaughtered in these establishments, where also the use of automated head-to-body electrical stunning is widespread.

\section{References}

Anil MH, McKinstry JL, 1998. Variations in electrical stunning tong placements and relative consequences in slaughter pigs. Vet J 155:85-90.

EFSA, 2004. Scientific report of the scientific panel for animal health and welfare on a request from the commission related to welfare aspects of animal stunning and killing methods. European Food Safety Authority ed., Parma, Italy.

European Commission, 1993. Council Directive of 22 December 1993 on the protection of animals at the time of slaughter or killing, 93/119/EEC. In: Official Journal, L 340, 31/12/1993.

European Commission, 2009. Council Regulation of 24 September 2009 on the protection of animals at the time of killing, 1099/2009/EC. In: Official Journal, L 303, 18/11/2009.

European Food Safety Authority, 2012. Scientific opinion. Statement on the use of animal-based measures to assess the welfare of animals. The EFSA Journal 10:2767.

Grandin T, 2010. Auditing animal welfare at slaughter plants. Meat Sci 86:56-65.

Grandin T, 2012a. Developing measures to audit welfare of cattle and pigs at slaughter. Anim Welfare 21:351-6.

Grandin T, 2012b. Recommended animal handling guidelines and audit guide. American Meat Institute Foundation ed., Washington, DC, USA. Available from: http:/www.animalhandling.org/ht/d/sp/i/26 752

Grandin T, 2013. Making slaughterhouses more humane for cattle, pigs, and sheep. Annu Rev Anim Biosci 1:491-512. 